\title{
HASTE INTRAMEDULAR DE POLIPROPILENO, COMBINADA OU NÃO A BIOMATERIAIS, NO TRATAMENTO DE FRATURAS INDUZIDAS NO ÚMERO DE POMBOS
}

(Use of polypropylene intramedullary rod alone or combined with biomaterials for treatment of induced humeral transverse fractures in pigeons)

\author{
TEIXEIRA, C.R. ${ }^{1}$; RAHAL, S.C. ${ }^{1}$; LIMA, A.F.M. ${ }^{1}$; CORREA, M.A. ${ }^{1}$; QUEIROZ, P. ${ }^{2}$; VULCANO, L.C. ${ }^{3}$
}

1Departamento de Cirurgia e Anestesiologia Veterinária;

${ }^{2}$ Médica Veterinária Autônoma;

${ }^{3}$ Departamento de Reprodução Animal e Radiologia Veterinária, Faculdade de Medicina Veterinária e Zootecnia (FMVZ)/UNESP, Campus de Botucatu, 18618-000, Rubião Júnior s/n - Botucatu, SP. Autor para correspondência; E-mail: sheilacr@fmvz.unesp.br.

RESUMO - O trabalho teve por objetivo avaliar a utilização da haste intramedular de polipropileno, combinada ou não a biomateriais de origem bovina, no tratamento de fraturas transversas induzidas no terço médio do úmero direito de pombos-domésticos. No grupo I $(n=7)$, a fratura foi estabilizada por meio de haste intramedular de polipropileno aplicada em ambos os fragmentos da fratura, sem ultrapassar as articulações. No grupo II $(n=7)$ utilizou-se a mesma fixação, porém foi aplicado junto ao foco de fratura uma mistura de biomateriais, constituída de proteínas morfogenéticas do osso e aglutinante de colágeno liofilizado. Em ambos os grupos, a asa foi imobilizada junto ao corpo com bandagem em forma de 8 por um período de 30 dias. Com seis semanas de pós-operatório, cinco fraturas do grupo I e quatro do grupo II estavam totalmente consolidadas. Foram observadas uma não consolidação hipertrófica (grupo I) e duas não consolidações com deslocamento do eixo ósseo (grupo II). Exceto as três aves que apresentaram complicações nas fraturas, as demais recuperaram a capacidade de vôo. Foi possível concluir que a haste intramedular de polipropileno foi um método de imobilização de eficácia limitada e os biomateriais utilizados não estimularam a consolidação das fraturas.

Palavras chaves: fratura, pombos, tratamento, cirurgia, ortopedia.

ABSTRACT - The aim of this study was to evaluate a polypropylene intramedullary rod alone or combined with biomaterials from bovine origin for the treatment of transverse fractures induced in the midshaft of right humerus in pigeons. In group I $(n=7)$ the fracture was immobilized using a polypropylene intramedullary rod inserted into both fractured extremities without entering the joints. The same device was used in group II $(n=7)$. Additionally, the fractured site in group II animals received a mixture of biomaterials composed by bone morphogenetic proteins and lyophilized collagen. In both groups a cast was applied to the affected wing, which was immobilized with a figure-of- 8 bandage applied to the body and wing during 30 days. Complete healing was observed after six weeks of surgery in five and four animals from groups I and II, respectively. Observed complications included one hypertrophic nonunion (group I) and two nonunion with bone shaft dislocation (group II). Except for the three pigeons that showed postoperative complications, the remaining animals recovered the ability of flight. It was possible to conclude that the polypropylene intramedullary rod is a limited method of immobilization and the biomaterials used in this study did not stimulate fracture healing.

Key words: fracture, pigeons, treatment, surgery. 


\section{Introdução}

As fraturas em aves apresentam alguns problemas que não são encontrados em mamíferos: primeiro, o osso é mais frágil, devido a alta densidade de cálcio e tende a esmigalhar ou quebrar mais facilmente; segundo, possuem ossos pneumáticos com espaços intramedulares grandes $(\mathrm{BUSH}$, 1986). Fraturas do úmero são comuns em aves e, geralmente, ocorrem nos terços médio e distal; as fraturas do terço médio têm bom prognóstico quando não há lesão muscular, do nervo radial ou do suprimento sangüíneo (MACCOY, 1992).

Diversos métodos de estabilização, como coaptação externa, pinos intramedulares metálicos ou não, aparelhos de fixação externa, resinas acrílicas, placas ósseas e parafusos, têm sido utilizados na dependência da localização e do tipo de fratura (REDIG, 1986a; BENNETT e KUZMA, 1992; WANDER et al., 2000). A decisão com relação aos implantes precisa ser baseada na necessidade da ave em retornar à capacidade de vôo ou apenas na estética e bem estar (REDIG, 1986a).

A cicatrização óssea, conforme BENNETT e KUZMA (1992), não é bem compreendida em aves; no entanto, quando não existe imobilização rígida e redução anatômica precisa ocorre consolidação óssea secundária. Clinicamente ossos de aves parecem cicatrizar mais rapidamente do que os de mamíferos.

As fraturas umerais são menos habilitadas ao manejo não cirúrgico, uma vez que tendem à instabilidade pela ação dos poderosos músculos flexores (REDIG, 1986a). O método mais consistente de tratamento, segundo REDIG (1986b), é a implantação de um pino intramedular com extremidade rosqueada e de tamanho suficiente para preencher dois terços da cavidade medular.

MACCOY (1983) afirmou que o pino intramedular de aço apresenta uma série de problemas no reparo de fraturas de ossos longos de aves voadoras. O peso do material pode impedir o vôo ou contribuir para injúrias auto-inflingidas, durante a consolidação da fratura, e as forças expansivas na colocação do pino podem causar fragmentação óssea. De acordo com BUSH (1986), aparelhos de metal colocados dentro ou ao redor do local de fratura enfraquecem a formação do calo endosteal e este, em aves, é o que mais contribui para a cicatrização.

A preocupação mais significante com relação ao uso de pino intramedular, segundo BENNETT e KUZMA (1992), é o possível dano as estruturas articulares e periarticulares. Quando este é colocado próximo à articulação pode causar deposição de tecido cicatricial que inibe a função articular normal. Contudo, quando aplicado no úmero, o pino tem menos chance de lesar as estruturas periarticulares, por causa do formato em S deste osso (MACCOY, 1983), devendo ser introduzido de forma retrógrada e saindo na crista deltóide (BENNETT e KUZMA, 1992).

BENNETT e KUZMA (1992) citaram que hastes de polímero de alta densidade e de polipropileno têm sido usadas isoladas, com fixador externo ou com polimetilmetacrilato. Estas hastes são de peso leve $(13 \%$ do peso do aço), facilmente esterilizadas e de baixo custo. Como as hastes de polipropileno proporcionam pouco controle de rotação, a estabilidade pode ser proporcionada com fios de Kirschner ou agulhas hipodérmicas colocados através da haste e corticais proximal e distal. Infelizmente, esta concentração de estresse nos pinos transversos predispõe à fratura óssea no orifício do pino. Outras formas de combater a rotação são a aplicação de um fixador externo, coaptação externa ou polimetilmetacrilato intramedular.

MACCOY (1983) utilizou um polímero de alta densidade na forma de haste, no reparo de fraturas umerais induzidas em seis pombos e em quatro fraturas espontâneas. O método proporcionou uma alternativa ao pino de aço em fraturas diafiseais simples, pois o leve peso do material fez sua remoção desnecessária, além do baixo custo.

As Proteínas Morfogenéticas Ósseas (BMPs), conforme RILEY et al. (1996), compreendem uma família de crescimento de mais de 12 proteínas, nove das quais têm mostrado individualmente induzir ossos 
Haste intramedular de polipropileno, combinada ou não a biomateriais, no tratamento de fraturas induzidas...

ectópicos em um sistema de prova In vivo. Elas parecem ter como alvo a célula perivascular tipo mesenquimal indiferenciada. Segundo LANE (2001), as BMPs são capazes de estimular células progenitoras a diferenciar e formar osso. Formas recombinantes de BMPs, particularmente BMP2, 4 e 7 têm capacidade de curar defeitos ósseos críticos em roedores, cães, carneiros e primatas, quando combinadas com um carreador de colágeno, matriz óssea desmineralizada, hidroxiapatita e polímeros biodegradáveis. MILLIS (1999) citou que as BMPs causam osteogênese de uma forma dose-dependente. Além disso, para atuarem durante a consolidação da fratura, elas precisam ser mantidas no local pela combinação a um carreador.

O trabalho teve por objetivo avaliar o efeito da haste intramedular de polipropileno, combinada ou não a biomateriais, no tratamento de fraturas induzidas no úmero de pombos.

\section{Material e Métodos}

Foram utilizados 14 pombos-domésticos (Columba livia), adultos, divididos por sorteio em dois grupos eqüitativos e identificados por meio de anilhas numeradas de 1 a 14. As aves foram confinados em gaiolas e receberam água e ração comercial ad libitum.

Para realização dos procedimentos cirúrgicos, os pombos foram sedados com levomepromazina $(1 \mathrm{mg} / \mathrm{kg})$ associado ao butorfanol $(0,1 \mathrm{mg} / \mathrm{kg})$ e midazolam $(0,5 \mathrm{mg} / \mathrm{kg})$ por via intramuscular, induzidos com cetamina (20 mg $/ \mathrm{kg} / \mathrm{lM})$, entubados e mantidos com anestesia inalatória com halotano.

As aves foram posicionadas em decúbito esternal e as penas da asa direita removidas. Após a anti-sepsia, realizou-se acesso dorsal por meio de incisão de pele longitudinal centrada sobre o úmero direito, exatamente caudal a margem do músculo bíceps. Posteriormente, o músculo bíceps foi afastado e os vasos maiores e nervo radial evitados. Foi induzida fratura transversa simples no terço médio do úmero direito, com o emprego de serra circular. No grupo I, a fratura foi estabilizada por meio de haste intramedular de polipropileno, originária de êmbolo de seringa descartável, aplicada em ambos os fragmentos da fratura e sem ultrapassar as articulações. No grupo II a fratura foi estabilizada da mesma forma que o grupo I, porém foi aplicado junto ao foco de fratura uma mistura de biomateriais, constituída de aglutinante colágeno de origem bovina liofilizado ${ }^{1}$ e proteínas morfogenéticas do osso adsorvidas à hidroxiapatita (BMPs) ${ }^{2}$. Os biomateriais foram misturados com solução fisiológica, nas proporções indicadas pelo fabricante, de forma a adquirir consistência pastosa. Com o foco de fratura reduzido, o produto foi aplicado ao redor das extremidades fraturadas. Em ambos os grupos, a fáscia superficial foi aproximada com uma sutura contínua simples e a pele com pontos isolados simples, ambos com fio náilon 4-0 agulhado. A asa foi imobilizada junto ao corpo em posição anatômica (bandagem em oito), por um período de 30 dias.

O cloranfenicol foi administrado na dose de $1 \mathrm{mg} /$ litro de água, por sete dias no período pós-operatório. Os pontos cutâneos foram removidos após dez dias. Radiografias foram efetuadas na posição ventrodorsal, após o procedimento cirúrgico e com seis semanas de pós-operatório.

Completados seis semanas de pósoperatório, as aves foram instaladas em viveiro com solário para reabilitação do vôo. Posteriormente foram soltas para vôo livre espontâneo em seu habitat natural, junto com outras aves não operadas, por um período de 60 dias. Ao final desse, realizou-se observação comparativa quanto à capacidade de vôo entre as aves operadas e não operadas.

\section{Resultados}

Na ave $n^{0} 1$ do grupo I ocorreu perda de um pequeno fragmento ósseo $(1 \mathrm{~mm})$ do foco de fratura, durante a secção óssea. Nas demais aves o procedimento cirúrgico ocorreu sem intercorrências. Nenhum pombo mostrou

${ }^{1}$ Gen-col - Baumer - Av. Pref. Antonio Tavares Leite, 181 - Mogi Mirim, SP.

${ }^{2}$ Gen-pro - Baumer - Av. Pref. Antonio Tavares Leite, 181 - Mogi Mirim, SP. 
TEIXEIRA, C.R. et al.

perda de apetite e todos toleraram bem a imobilização externa da asa com esparadrapo. Além disso, as feridas cirúrgicas cicatrizaram sem complicações.

A avaliação radiográfica pós-operatória imediata mostrou alinhamento do foco de fratura, sem desvio do eixo ósseo na maioria das aves. A exceção foi o pombo $n^{\circ} 5$ do grupo I, que apresentava afastamento das extremidades ósseas. Encontra-se na tabela
1 o resultado final da evolução das fraturas dos pombos dos grupos I e II, respectivamente. $\mathrm{Na}$ palpação do foco de fratura, com seis semanas de pós-operatório, não se verificou mobilidade ou crepitação, exceto nas aves que apresentaram deslocamento do foco de fratura ( $n^{\text {os }} 8$ e 10$)$.

De um total de 14 aves, três não recuperaram a capacidade de vôo, sendo uma do grupo I $\left(\mathrm{n}^{\circ}\right.$ $5)$ e duas do grupo II (nos 8 e 10).

TABELA 1 -AVALIAÇÃO RADIOGRÁFICADAS FRATURAS DOS POMBOS PERTENCENTES AO GRUPO I (NOS 1 A 6) E GRUPO II (NOS 7 A 14) COM SEIS SEMANAS DE PÓS-OPERATÓRIO. BOTUCATU, 2003.

\begin{tabular}{|c|c|}
\hline Pombo $\left(n^{\circ}\right)$ & Avaliação radiográfica \\
\hline 1 & fratura consolidada, moderado calo externo face medial \\
\hline 2 & fratura consolidada, discreto calo externo face medial (FIGURA 1) \\
\hline 3 & $\begin{array}{l}\text { fratura não totalmente consolidada, com calo externo exuberante, sem perda de } \\
\text { alinhamento ósseo }\end{array}$ \\
\hline 4 & $\begin{array}{l}\text { fratura consolidada, com moderado calo externo medial e discreto cisalhamento } \\
\text { horizontal }\end{array}$ \\
\hline 5 & não consolidação hipertrófica \\
\hline 6 & $\begin{array}{l}\text { fratura consolidada, moderado calo externo face medial, discreto cisalhamento } \\
\text { horizontal }\end{array}$ \\
\hline 7 & fratura consolidada, calo externo moderado lateral e discreto medial \\
\hline 8 & fratura não consolidada com deslocamento do eixo ósseo \\
\hline 9 & $\begin{array}{l}\text { fratura consolidada, moderado calo externo face medial, discreto cisalhamento } \\
\text { horizontal }\end{array}$ \\
\hline 10 & fratura não consolidada e com grave deslocamento do eixo ósseo \\
\hline 11 & fratura consolidada, moderado calo externo nas faces medial e lateral \\
\hline 12 & fratura não consolidada, exuberante calo externo medial \\
\hline 13 & fratura consolidada, discreto calo externo medial \\
\hline 14 & fratura consolidada, moderado calo externo nas faces medial e lateral (FIGURA 2) \\
\hline
\end{tabular}

FIGURA 1 - RADIOGRAFIA VENTRODORSAL DO POMBO № 2, PERTENCENTE AO GRUPO I, SEIS SEMANAS APÓS O PROCEDIMENTO CIRÚRGICO. OBSERVE A FRATURA DO ÚMERO DIREITO CONSOLIDADA (SETA) E COM DISCRETO CALO EXTERNO FACE MEDIAL.

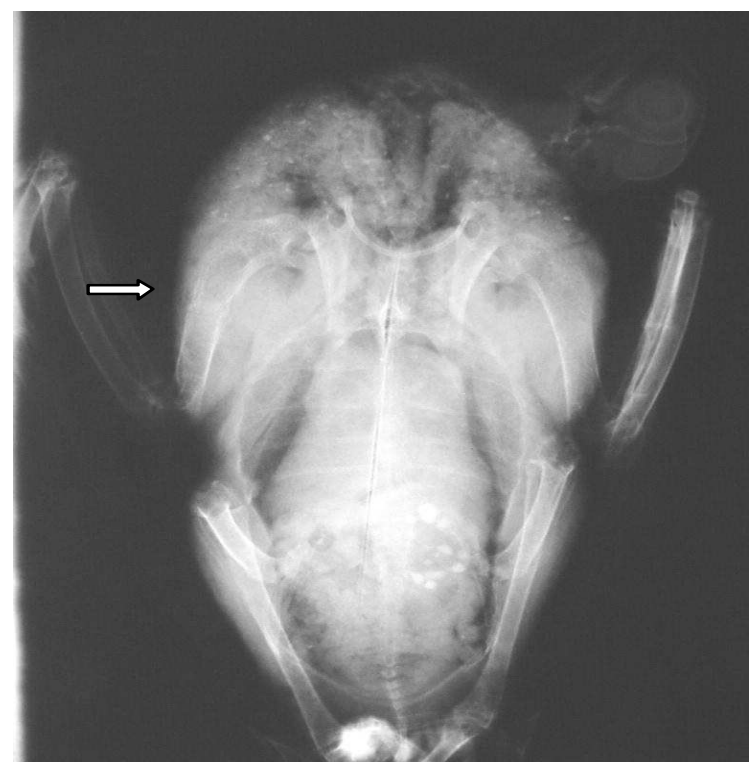


Haste intramedular de polipropileno, combinada ou não a biomateriais, no tratamento de fraturas induzidas...

FIGURA 2 - RADIOGRAFIA VENTRODORSAL DO POMBO N 14, PERTENCENTE AO GRUPO II, SEIS SEMANAS APÓS O PROCEDIMENTO CIRÚRGICO. NOTE A FRATURA CONSOLIDADA (SETA) COM MODERADO CALO EXTERNO NAS FACES MEDIAL E LATERAL.

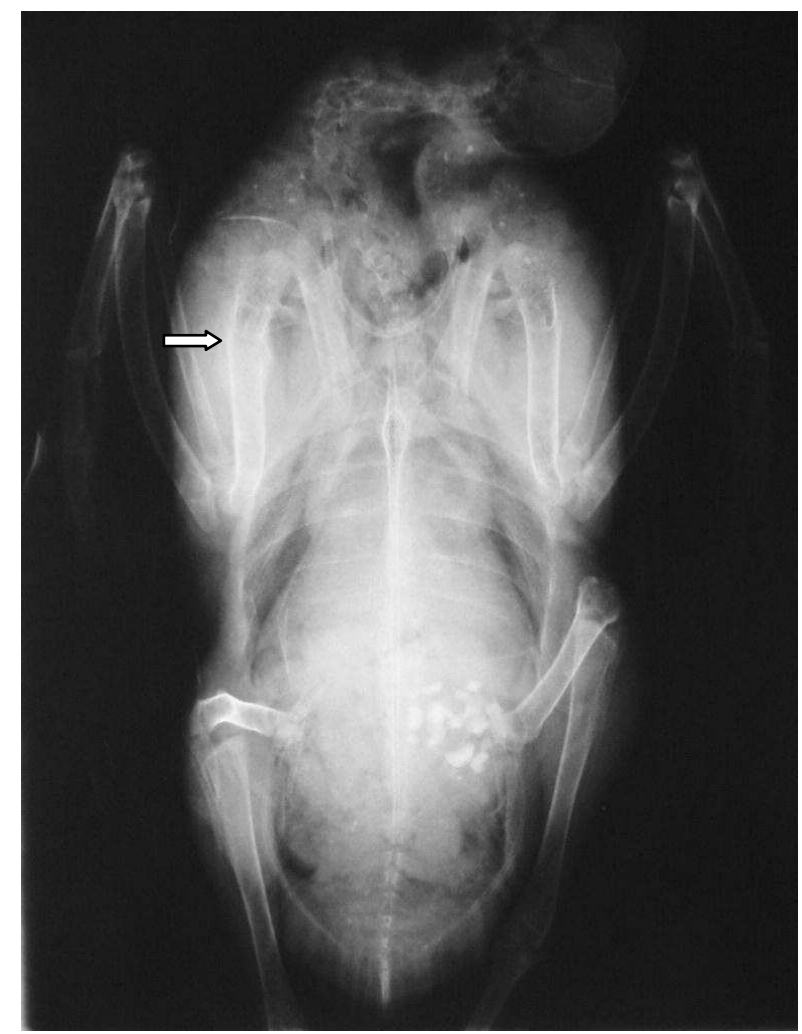

\section{Discussão}

A haste intramedular de polipropileno, como referido por MACCOY (1983) e BENNET e KUZMA (1992), apresentou vantagens como baixo custo e leveza. Entretanto, apesar desse implante apresentar saliências externas, não teve capacidade de controlar o movimento de rotação da fratura, em especial pela largura do canal medular, que é uma das características do osso de aves (BUSH, 1986), bem como pela dificuldade de fixação nas extremidades. O fato também é observado com o pino de aço de Steinmann, que, além disso, apresenta problemas relacionados ao peso, possibilidade de fragmentação óssea (MACCOY, 1983), e lesão às estruturas articulares e periarticulares (BENNET e KUZMA, 1992).

Entre os diversos métodos citados (REDIG, 1986b; BENNETT e KUZMA, 1992) para combater a rotação, optou-se pela coaptação externa da asa com esparadrapo. O método foi de fácil aplicação e confortável; contudo, não foi suficiente para controlar a rotação em $21,42 \%$ dos animais. Como no pósoperatório imediato, com exceção da ave $\mathrm{n}^{\circ}$ 5 do grupo I, todas as fraturas estavam alinhadas, supõe-se que ocorreu afrouxamento da coaptação durante o período pós-operatório, favorecendo o deslocamento ósseo. Dessa forma, observa-se que a bandagem externa é fundamental para que ocorra adequada evolução do foco de fratura.

Mesmo nos casos em que a fratura consolidou sem complicações, havia formação de calo externo, em sua maioria discreto ou moderado, indicando consolidação óssea secundária que é, como citado por BENNETT e KUZMA (1992), característica de imobilização não rígida.

Apenas pela avaliação radiográfica não foi possível estabelecer diferenças entre o grupo I e o grupo II (biomateriais). Como a BMP utilizada não é recombinante, mas sim um "pool", a capacidade de estimulação de células progenitoras pode ser inferior a 
observada com as BMP 2, 4 e 7 (JOSEPH, 2001). Apesar do produto vir adsorvido a um carreador de hidroxiapatita que, conforme MILLIS (1999), é necessário para manutenção das BMP, não foi utilizado membrana osteopromotora. Dessa forma, o tempo de permanência das BMPs pode não ter sido suficiente para estimular a diferenciação celular. Por outro lado, denotouse que o biomaterial não interferiu negativamente, já que os tipos de complicações observadas no grupo II se assemelharam as do grupo I e estavam associadas ao controle inadequado das forças da fratura pelo método de imobilização adotado.

A capacidade de vôo foi observada apenas nas aves em que as fraturas consolidaram sem deslocamento do alinhamento do eixo ósseo, sendo dessa forma fundamental para o retorno funcional. De forma semelhante, REDIG (1986c) referiram que após o tratamento cirúrgico de fraturas umerais em 51 aves, a consolidação ocorreu em 18 e a capacidade de vôo foi alcançada em apenas 12.

\section{Conclusões}

Foi possível concluir que a haste intramedular de polipropileno é um método de imobilização de eficácia limitada e os biomateriais utilizados não estimularam a consolidação das fraturas.

\section{Referências}

BENNETT, R.A.; KUZMA, A.B. Fracture management in birds. Journal of Zoo and Wildlife Medicine, Lawrence, v.23, n.2, p.5-38, 1992.

Recebido para publicação: 27/12/2003 Aprovado:

$05 / 04 / 2004$
BUSH, M. Laparoscopy and surgery. In: FOWLER, M.E. Zoo \& Wild Animal Medicine. 2.ed. Philadelphia: W.B. Saunders, 1986. p.254261.

LANE, J.M. BMPs: why are they not in everyday use? Journal of Bone Joint Surgery American, Neidhan, v.83, p.161-162, 2001.

MILLIS, D.L. Bone and non-bone - derived growth factors and effects on bone healing. Veterinary Clinics of North America: Small Animal Practice, Philadelphia, v.29, p.1221-1246, 1999.

MACCOY, D.M. Treatment of fractures in avian species. Veterinary Clinics of North America: Small Animal Practice, Philadelphia, v.22, p.225238, 1992.

REDIG, P.T. Evaluation and nonsurgical management of fractures. In: HARRISON, G.J.; HARRISON, L.R. Clinical Avian Medicine and Surgery. Philadelphia: W.B. Saunders, 1986a. chap. 30, p.380-394.

REDIG, P.T. Basic orthopedic surgical techniques. In: HARRISON, G.J.; HARRISON, L.R. Clinical Avian Medicine and Surgery. Philadelphia: W.B. Saunders, 1986b. chap.49, p.596-598.

REDIG, P.T. A clinical review of orthopedic techniques used in the rehabilitation of raptors. In: FOWLER, M.E. Zoo \& Wild Animal Medicine. Philadelphia: W.B. Saunders, 1986c. p.388-401.

RILEY, E.H.; LANE, J.M.; URIST, M.R.; LYONS, K.M.; LIEBERMAN, J.R. Bone morphogenetic protein-2. Clinical Orthopaedics and Related Research, Hgerstok, n.324, p.39-46, 1996.

WANDER, K.W.; SCHWARZ, P.D.; JAMES, S.P.; POWERS, B.E.; TAYLOR, B.; WIMSATT, J.H. Fracture healing after stabilization with intramedullary xenograft cortical bone pins: a study in pigeons. Veterinary Surgery, Philadelphia, v.29, p.237-244, 2000. 